\title{
Topic Study Group No. 1: Early \\ Childhood Mathematics Education \\ (Up to Age 7)
}

\author{
Elia Iliada, Joanne Mulligan, Ann Anderson, Anna Baccaglini-Frank \\ and Christiane Benz
}

\section{The Programme}

\section{Session 1 papers}

- Nathalie Sinclair: Time, immersion and articulation: Digital technology for early childhood mathematics

- Iliada Elia: Gestures and their interrelations with other semiotic resources in the learning of geometrical concepts in kindergarten

- Jennifer Thom: Circling three children's spatial-geometric reasonings

Session 2 papers

- Gina Bojorque: Ecuadorian kindergartners' SFON development (presented by Joke Torbeyns)

- Sanne Rathé: Kindergartners' spontaneous focus on number during picture book reading

- Christiane Benz: Measurement makes numbers sensible

Session 3 papers

- Joanne Mulligan: Promoting early mathematical structural development through an integrated assessment and pedagogical program

Co-chairs: Elia Iliada, Joanne Mulligan.

Team members: Ann Anderson, Anna Baccaglini-Frank, Christiane Benz.

E. Iliada $(\square)$

University of Cyprus, Nicosia, Cyprus

e-mail: elia.iliada@ucy.ac.cy; iliadaelia@gmail.com

J. Mulligan

Macquarie University, Sydney, Australia

e-mail: joanne.mulligan@mq.edu.au

(C) The Author(s) 2017 
- Miriam M. Lüken: Repeating patterning competencies in 3- and 4-year old kindergartners

- Ralf Kampmann: The influence of fostering children's pattern and structure abilities on their arithmetic skills in Grade 1

- Ruthi Barkai: Preschool teachers' responses to repeating patterns tasks

Session 4 papers

- Ann Anderson: A study of types of math-in-context that parents and preschoolers share at home

- Herbert P. Ginsburg: Interactive mathematics books and their friends

- Anna Baccaglini-Frank: Educational multi-touch applications, number sense, and the homogenizing role of the educator

TSG 1 included research-based contributions on recent trends and developments in early mathematics learning and teaching which stimulated rich discussions and enabled a deep understanding on various important issues in the field of early childhood mathematics education. As indicated in the programme, the wide scope of the studies reported in the presentations were organized into four sessions.

The focus of the first session of TSG 1 was on multimodal, embodied and semiotic aspects of learning with or without technology. The contribution by Sinclair discussed three innovative issues on the use of digital technology in early mathematics education that may transform the learning and teaching of mathematics. The first theme referred to temporalizing early childhood mathematics (time), which suggested that the use of dynamic geometry software and a multi-touch App for counting through embodied actions highlights dynamic aspects of mathematical objects, which promote the learning of sophisticated mathematical ideas. The second aspect concerned children's contact with advanced mathematics (immersion) often beyond the curriculum, because of the less constrained digital environments. The third issue was about the affordance of digital technology to support the articulation of signs in children's mathematical work.

The case study presented by Elia focused on the links between gestures and other semiotic resources in the understanding of geometrical concepts and the changes in these interrelations as learning evolves. Analyzing longitudinal observation data from a kindergarten class, and specifically from a child while interacting with teacher and peers, showed that gestures together with oral language and semiotic inscriptions had a major role in the kindergartner's development of geometric awareness for 2D shapes, their attributes and also in the process of shape dimensional deconstruction. As the child's geometry thinking evolved, his words and gestures were detached from the materiality of the activities and were based on the use of imaginary and general geometrical objects.

The contribution by Thom gave further insight into young children's spatial-geometric reasoning by elucidating the role of embodied actions in children's work. As part of a larger research project, Thom analyzed the forms, acts and processes that constitute children's reasoning while working on a spatial-geometric task. A photograph of a cylinder elicited different mathematical ideas and ways of 
reasoning in three grade one children. The study showed how these were materialized as gestures, movements, drawings, imagery and verbalizations. Another important finding of the study was the generative co-emergence between the children's reasoning and spatial-geometric conceptions, involving the transition between multiple dimensions, visualisation, decomposition, re-composition, perspective taking, dynamic objects, rotation, symmetry, curved and flat surfaces.

In the second session of TSG 1 different aspects of arithmetical competences of children built the thematic connection of the three presentations. Two of the presentations focused on the SFON effect: the Spontaneous Focusing On Numerosity. The first study was conducted with kindergarten children in Ecuador by Bojorque and presented by Torbeyns. Considering that SFON studies reported a positively associated SFON tendency to the development of early numerical abilities, Bojorque reported on a study where children were tested with SFON tasks at the beginning and end of a kindergarten year and on early numerical abilities at the beginning of the year. Findings showed that there was a limited SFON development and a positive relation between SFON and early numerical abilities. The quality of early mathematics education in Ecuador did not contribute to kindergartners' SFON development.

In the presentation by Rathé the role of picture books and a possible association to the children's SFON was investigated. The study was conducted in Belgium with children at kindergarten age. It examined the association between children's SFON in experimental tasks and number related utterances during everyday picture book reading. No correlation was found between these aspects. Various hypothetical explanations were given for this result.

The relation between measurement and number development was the theme in the presentation by Benz and Pullen which reported on a study with children at ages 5-6 in Australia. A design research was presented where children started formal schooling at a school with a Reggio Emilia and socio-cultural approach. The children did not start with a typical number-focused curriculum but with a measurement-focused curriculum where number concepts were included. Especially the case studies revealed that when young children measure, they use numbers and can acquire number competencies.

During the third session of TSG 1, there were four presentations focusing on patterning and structural competencies for preschoolers through to formal schooling as well as for preschool teachers. Mulligan provided an overview of the Australian Pattern and Structure project comprising a suite of studies with 4-8 year olds. She provided examples of children's Awareness of Mathematical Pattern and Structure (AMPS), a new construct which had been identified and measured, and found to be predictive of general mathematical achievement. An assessment interview, the Pattern and Structure Assessment (PASA) and Learning Pathways of the Pattern and Structure Mathematical Awareness Program (PASMAP) were illustrated.

Similarly, Lüken focused on the development of repeating patterning abilities in early math learning. As part of a longitudinal exploratory study, she analyzed the responses of individual children's patterning from their first to third year of German kindergarten. Three interviews, spanning two years, were presented, suggesting that 
significant development takes place in children's patterning competencies between the age of three and four. She described two important stages: the abilities to refer to the existing pattern and to alternate two colors.

In a related paper, Kampmann showed that patterning and structural abilities can positively influence arithmetic skills in Grade 1 . He described an intervention study with 51 first-graders, showing significant differences between pre- and post-test arithmetic achievement scores for the intervention group. The improvement was particularly beneficial for the lowest achieving children. The intervention lessons included recognizing, describing, explaining and creating patterns with an emphasis on structuring the base 10 system.

The presentation by Barkai presented the work of her team (Pessia Tsamir, Dina Tirosh, Esther Levenson, and Michal Tabach) on a study of preschool teacher knowledge, and the important role of the preschool teachers and their ability to identify and continue repeating patterns. Their study found that preschool teachers were able to identify drawings which represent repeating patterns and find the errors which preclude a drawing from actually being a repeating pattern. However, identifying appropriate continuations was more difficult. They highlighted the use of the Cognitive Affective Mathematics Teachers Education (CAMTE) framework as a research tool and the importance of investigating teachers' knowledge of producing and evaluating solutions.

During the final session of TSG 1, three presenters shared their research into preschoolers' mathematical learning within everyday parent-child mathematical interactions, interactive mathematical books produced for parent or teacher-child shared reading, and teacher-child interactions around two iPad apps, respectively. In the observational study she presented, Anderson and Anderson reported on the types of mathematics that six preschoolers shared with family members during 'naturally occurring' activities that each of their middle class mothers chose to have videotaped. Across the dyads, a range of mathematics concepts were found; with one family sharing mostly number-related activities, while four other families shared more geometry-related activities. Likewise, although activities appeared common across the families, the nature of the specific materials, and/or the specific adult-child interactions oftentimes meant, the mathematics shared within these particular contexts differed.

In his presentation, Ginsburg focused on key features of the digital resource he and his team designed and produced. As Ginsburg indicated, interactive mathematics books, fiction and non-fiction, enveloped in a digital surround of supporting materials - their "Friends" - can delight and educate young children as well as those (e.g., parents, teachers, siblings) who read with them, yet few such books now exist, and little is known about them. In his presentation, he described, and illustrated, the potential of interactive mathematics storybooks (IMS), which entail a special set of affordances that can promote young children's mathematics learning, and the surrounding Friends, which can help the adult understand the mathematics and the child.

The use of digital tools to support and possibly enhance adult-child joint activity was a theme of the final presentation, where Baccaglini-Frank reported on her study 
of teacher-child interactions. As part of an educational project proposed in Italian preschools, an educator followed a tested protocol proposing two chosen iPad apps to children of ages 5-6, in which interactions with the software occur through multiple-touch gestures on the screen. Though, the educator's interventions were supposedly aimed at strengthening the children's number sense, the result was a homogenization of their schemes, in various cases seemingly inhibiting development of number sense.

Overall TSG 1 drew attention to contemporary and integrated perspectives on mathematics learning and teaching in the early years based on research studies from around the world and contributed to the ongoing discussion on how to advance research, development and practice in early childhood mathematics.

Open Access Except where otherwise noted, this chapter is licensed under a Creative Commons Attribution 4.0 International License. To view a copy of this license, visit http://creativecommons. org/licenses/by/4.0/.

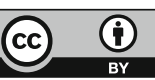

